\title{
Philippe RIVIALE, Gracchus Babeuf, Robespierre et les tyrans suivi de Du système de dépopulation ou la vie et les crimes de Carrier par Gracchus Babeuf
}

\section{Claude Mazauric}

\section{(2) OpenEdition}

1 Journals

\section{Édition électronique}

URL : https://journals.openedition.org/ahrf/12383

DOI : $10.4000 /$ ahrf.12383

ISSN : 1952-403X

Éditeur :

Armand Colin, Société des études robespierristes

\section{Édition imprimée}

Date de publication : 1 mars 2012

Pagination : 223-226

ISSN : 0003-4436

\section{Référence électronique}

Claude Mazauric, "Philippe RIVIALE, Gracchus Babeuf, Robespierre et les tyrans suivi de Du système de dépopulation ou la vie et les crimes de Carrier par Gracchus Babeuf ", Annales historiques de la Révolution française [En ligne], 367 | janvier-mars 2012, mis en ligne le 12 septembre 2012, consulté le 01 juillet 2021. URL : http://journals.openedition.org/ahrf/12383 ; DOI : https://doi.org/10.4000/ahrf.12383

Ce document a été généré automatiquement le 1 juillet 2021.

Tous droits réservés 


\title{
Philippe RIVIALE, Gracchus Babeuf, Robespierre et les tyrans suivi de Du système de dépopulation ou la vie et les crimes de Carrier par Gracchus Babeuf
}

\author{
Claude Mazauric
}

\section{RÉFÉRENCE}

Philippe RIVIALE, Gracchus Babeuf, Robespierre et les tyrans suivi de Du système de dépopulation ou la vie et les crimes de Carrier par Gracchus Babeuf, Paris, L'Harmattan, 2011, 369 p., ISBN 978-2-296-54204-4, $33 €$.

1 Auteur d'ouvrages consacrés à Babeuf et aux Égaux en leur temps remarqués, notamment par François Hincker en 1995 et par Raymonde Monnier en 2002 dans les Annales historiques de la Révolution française, Philippe Riviale réédite aujourd'hui le fameux Du système de dépopulation [...], 194 pages, paru au début de l'an III chez l'imprimeur Franklin, rue de Cléry à Paris. Philippe Riviale fait précéder la réédition du texte de Babeuf d'une profuse introduction de 257 pages et l'accompagne d'un addendum de quatre pages, extrait de la Dénonciation faite aux comités de salut public et d'agriculture de la Convention, d'un complot de famine contre Paris, indiquée comme datant du 18 juillet 1793 quand François-Noël Babeuf était employé dans l'administration des subsistances de Paris (la référence donnée : "Source Gallica », est imprécise mais selon l'Inventaire des manuscrits et imprimés de Babeuf (B.N. 1976) le document cité pourrait concerner la pièce $\mathrm{BN} 8^{\circ} \mathrm{Lb} 41$ 752).

Une réédition du livre de Babeuf $D u$ système de dépopulation [...] avait déjà paru chez Tallandier à l'initiative de Reynald Sécher et Jean-Joël Brégeon, lesquels, au moment du bicentenaire, avaient prétendu embarquer Babeuf parmi les dénonciateurs du "génocide franco-français » proposé par Pierre Chaunu. En son temps, j’avais dénoncé 
la manœuvre dans un article ( Sur Babeuf à propos de la Vendée » Regards sur les sociétés modernes, Mélanges offerts à Claude Petitfrère, Tours, 1997, p. 251-264) en replaçant dans sa configuration historique et biographique, le contenu de l'ouvrage de Babeuf, rédigé, je le rappelle, au cours de l'automne thermidorien quand se profilait à Paris le procès intenté à Carrier. En 2008, aux Editions du Cerf, Reynald Sécher et Jean-Joël Bourgeon ont relancé une nouvelle réédition du texte de Babeuf, cette fois-ci préfacée par Stéphane Courtois, lequel cherche à embrigader le « communiste » Babeuf contre lui-même, parmi les précurseurs de la réflexion sur les populicides parce qu'il aurait " analysé avec finesse la tentative révolutionnaire d'exterminer la Vendée ». Dans le livre dont il est fait ici recension, Philippe Riviale s'inscrit en faux avec justesse et courage contre cette prétention dont il observe plus qu'il ne les analyse d'ailleurs, les présupposés idéologiques mais en s'évertuant, non sans succès, à en montrer l'inanité conceptuelle et factuelle: c'est le mérite principal de la longue "première partie: Babeuf dans la Révolution» qui tient lieu d'introduction au texte même de Babeuf, lequel n'occupe que le tiers de la pagination du livre tout entier. En elle-même, cette appréciable réfutation sera remarquée par les spécialistes de la période, en raison de l'intérêt nouveau que suscite l'ouvrage de Babeuf. L'article de Ronan Chalmin, «La république populicide: relire Du système de dépopulation de G. Babeuf », paru dans la dernière livraison de la revue XVIII e siècle $\left(\mathrm{n}^{\circ} 43,2011\right.$, pp. 449-468) montre bien le renouveau d'intérêt pour ce texte.

3 La première partie du livre de Philippe Riviale se présente donc comme un long essai d'histoire de la Révolution. Cette sorte d'introduction profuse, juxtapose en quinze chapitres successifs d'inégale longueur, divers développements; mais ceux-ci fort souvent n'ont pas de rapports directs avec l'élaboration du texte de Babeuf lui-même : ainsi du chapitre portant comme titre «De la force du gouvernement actuel de la France et de la nécessité de s'y rallier (p. 93-98) ", six pages où il est question de la brochure bien connue de Benjamin Constant parue après le texte de Babeuf, lors de l'avènement du Directoire, et donc sans effet possible sur l'élaboration du Système de dépopulation sauf à s'embarquer dans une sorte de méta-histoire éloignée de la préoccupation des historiens proprement dits. Les rapports de Babeuf avec le mouvement de la Révolution avant Thermidor sont abordés ici ou là sous un éclairage délibérément anachronique : ainsi l'analyse des « idées de Babeuf » relatives à la notion d'égalité est-elle introduite par une réfutation de l'idée selon laquelle elles auraient eu quelque rapport avec la conception du "partage» qui, "de l'Icarie de Cabet au socialisme réel, où le système des faveurs, de l'intrigue et des supériorités régna sous couvert des mots d'ordre, auxquels personne ne crut, après peu de temps » (p. 109) s'est par la suite répandue... Quelle serait donc la conception de l'égalité de Babeuf? «La dignité de la vie pour chacun»! Voilà assurément qui est un peu court pour comprendre la haine de classe que Babeuf suscita de son vivant et que l'auteur d'ailleurs, qui aime Babeuf, stigmatise en permanence... L'anachronisme qui caractérisait souvent les ouvrages précédents de Philippe Riviale, ouvrages auxquels il renvoie fréquemment le lecteur pour se dispenser de pousser ses démonstrations ( $L a$ conjuration, 1994, L'impatience du bonheur, 2001), présente quelquefois des aspects surprenants. Ainsi lit-on, par exemple, sous sa plume : «On ne sera pas étonné, si je rappelle que l'« hostilité aux partis » de Babeuf, selon la supposition de l'auteur, fut celle-là même qu'éprouva Simone Weil » dans ses Ecrits de Londres parus en 1957 ! Une comparaison appuyée de longues citations de Weil présentées sans commentaire, comme si la notion de "partis" français au $\mathrm{xx}^{\mathrm{e}}$ siècle était comparable à celle de 
«partis » comme Babeuf et ses contemporains les entendaient en 1794 ! L'affirmation est suivie sans transition d'une évocation de la misère française inspirée des récits de voyage d'Arthur Young en France en 1788, 1789 et 1792, elle-même suivie de ce constat baroque : «Que ce soit en Indochine en 1946, en Algérie en 1954, il en fut comme pour la guerre de Vendée... ». Vient alors en conclusion du développement, une citation de Babeuf tirée du Système de dépopulation supposée apporter démonstration des vérités antérieurement énoncées. A quoi peut conduire une telle rhétorique superficielle? S'agissant de l'histoire de la Révolution française comme processus ou succession d'événements, j'avoue n'avoir pas réussi à me faire une idée d'ensemble de ce qui fonde la doxa proposée par Philippe Riviale dans sa longue dissertation: il est question de "tyrans" et de "tyrannie», de corruption et d'«oppresseurs", d'ambition et d'« argent conspirateur», de "Carrier, monstre ordinaire», de "tyrans nouveaux après Thermidor " ... auxquels s'opposent de « nouveaux rebelles », mais, en se fondant sur une lecture attentive, on éprouve, du moins m'a-t-il semblé, des difficultés quasiinsurmontables à comprendre ce qui a mis en mouvement la France à la fin du XVIII ${ }^{\mathrm{e}}$ siècle : du coup la place de Babeuf dans ce dispositif confus, n'est guère autre que celle d'un prophète dont la parole se situe finalement hors du temps, hors de son temps : on ne saurait mieux régresser en amont de l'œuvre de Victor Daline (pour ne retenir ici que le nom de l'historien qui a renouvelé en profondeur la connaissance de Babeuf) qui montre Babeuf héritier et innovateur dans la pratique politique, du projet ancestral de construction d'une nouvelle société faite pour tous les humains, non pour des minorités de dominants exploiteurs. Robespierre comme Saint-Just, en tant qu'individus, se sortent assez bien des jugements souvent formulés à l'emporte-pièce par Philippe Riviale dans les cinq premiers chapitres et dans le douzième de la "première partie " mais en tant que protagonistes d'une transformation démocratique de l'institution politique et comme penseurs de la «Terreur» en tant qu'instrument transitoire du gouvernement, rien, à mon sens, ne nous est dit de cohérent, donc de convaincant. Or la compréhension du Système de dépopulation de Babeuf dont le contenu vise à faire comprendre que sans établissement d'une transformation sociale radicale, à quoi ont renoncé, selon lui, ceux que nous appelons les "robespierristes", une seule voie se dégage, celle de la tyrannie de la propriété particulière et de l'accaparement des richesses par les oligarchies, orientation qui a conduit (et conduira) au mal-être social, à la violence tyrannique, au populicide et à la régression humaine, ces maux absolus contre lesquels se dresse déjà celui qui va bientôt se dire le " Tribun du peuple ».

4 Soulignons enfin que l'auteur du livre ne se prive jamais de régler des comptes dont on voit mal l'origine ou l'intérêt, à moins d'être soi-même informé de circonstances dont l'histoire est obscure : du moins l'est-elle pour l'auteur de ce compte rendu. Ainsi, à la page 20, Riviale exécute-t-il, de manière insupportable, l'excellente étude, novatrice en son temps, de Françoise Brunel («Mélanges sur l'historiographie de la réaction thermidorienne; pour une analyse de l'échec de la voie jacobine ", AHRF, 1979) où il ne voit que " confusion", « erreur de jugement ", reprise de «l'historiographie usuelle de Mathiez à Soboul », etc. A la page 107, une note 2 nous apprend ceci : « Quand j'étudiais à la Faculté de droit de Paris (...) j'eus une algarade avec M. Jean-Louis Debré -l'actuel président du conseil constitutionnel, (NDR) - mon condisciple, pour avoir protesté (contre l'emploi des mots « gueux, canaille, populace » NDR). J'étais, faut-il le dire, seul à le faire dans cette aimable société d'élite » : en quoi cette confidence nous aide-t-elle à saisir le contexte de la parution du Système de dépopulation de Babeuf ? Page 113 (note 2), Philippe Riviale tient avec juste raison à se démarquer de l'article bien connu et 
survalorisé en son temps de M. Andrews (Annales ESC, 1974, p. 73-106) : mais pourquoi l'accompagner de ce commentaire : «Pauvre Marc Bloch, pauvre Lucien Febvre » [...] «Andrews admire les vainqueurs qui sont habiles et méprise les perdants qui ne savent pas s'adapter, etc. ». Et d'ajouter perfidement : "J'ai fait justice dans La Conspiration de l'article de M. Andrews, dont l'heure de gloire était venue avec la reconnaissance des pairs en Histoire considérée selon la chirurgie esthétique. Pour couronner l'effet, il y eut le pâle acquiescement de Claude Mazauric, ci-devant tenant du titre de «spécialiste de Babeuf " qui parla de front populaire avec les ex-conventionnels Amar, Vadier, Laignelot et Co. Puis vint François Furet, Napoléon de l'EHESS ». Sans alourdir la barque, je ferai remarquer à $M$. Riviale que ma critique sévère de l'article de Andrews qui n'avait donc rien d'un acquiescement - était, elle, respectueuse de l'homme et du chercheur, et bien antérieure à la sienne, datant de la réédition de mon recueil Babeuf. Ecrits en 1988, qu'il a toujours l'air d'ignorer même après sa réédition récente, en 2009 au Temps des cerises, un recueil augmenté, corrigé et complété, signalant en outre dans la bibliographie les ouvrages de l'auteur ici en question! Quant à l'amalgame opéré in fine entre feu Furet et ma modeste personne, laquelle n'a rien de celle d'un mentor et se montre encore vivante, il surprendra celles et ceux qui n'ignorent rien de l'histoire intellectuelle récente en France (et ailleurs)!

5 Reste que le livre de Philippe Riviale, en ce qu'il nous propose une réédition bien venue de l'ouvrage thermidorien de Babeuf, intéressera tous ceux qui tiennent l'histoire de Babeuf et des babouvistes pour une grande histoire, toujours en débat. Et j'ajouterai pour ma part que son auteur aura beau s'y évertuer, il n'arrivera pas à faire du signataire de ce bref compte-rendu son adversaire. Pas plus aujourd'hui que lors du Colloque de Vendôme de 1997 dont les Actes ont paru et où nous étions présents de concert avec Michel Vovelle et quelques autres! 